\title{
Mapping and Evaluation of Malus xdomestica Microsatellites in Apple and Pear
}

\author{
Minou Hemmat \\ Department of Horticultural Sciences, Cornell University, New York State Agricultural Experiment Station, \\ Geneva, NY 14456 \\ Norman F. Weeden \\ Department of Plant Sciences and Plant Pathology, Montana State University, Bozeman, MT 59717 \\ Susan K. Brown \\ Department of Horticultural Sciences, Cornell University, New York State Agricultural Experiment Station, \\ Geneva, NY 14456
}

AdDITIONAL INDEX WORDS. SSR, Malus, linkage map, Pyrus communis

\begin{abstract}
We mapped DNA polymorphisms generated by 41 sets of Simple Sequence Repeat (SSR) primers, developed independently in four laboratories. All primer sets gave polymorphisms that could be located on our 'White Angel' $x$ 'Rome Beauty' map for apple [Malus sylvestris (L.) Mill. Var. domestica (Borkh.) Mansf.]. The SSR primers were used to identify homologous linkage groups in 'Wijcik McIntosh', NY 75441-58, 'Golden Delicious', and 'Liberty' cultivars for which relatively complete linkage maps have been constructed from isozyme and Random Amplified Polymorphic DNA (RAPD) markers. In several instances, two or more SSRs were syntenic, and except for an apparent translocation involving linkage group (LG) 6, these linkages were conserved throughout the six maps. Twenty-four SSR primers were consistently polymorphic, and these are recommended as standard anchor markers for apple maps. Experiments on a pear (Pyrus communis L.) population indicated that many of the apple SSRs would be useful for mapping in pear. However some of the primers produced fragments in pear significantly different in size than those in apple.
\end{abstract}

Simple sequence repeats (SSRs), also called microsatellites, consist of different numbers of tandem repeats (two to six nucleotides in length) and have a total length of repeated units in the range of 10 to $60 \mathrm{bp}$. SSRs are highly informative genetic markers and have been used in saturation and construction of high-density maps of human and other mammalian systems. Based on properties such as co-dominant mode of inheritance and high degree of heterozygosity, SSRs have advantages over other types of markers. In plants, SSRs have been reported to be highly polymorphic and provide many alleles for each primer set in closely related species. Such markers are particularly useful in species with low genetic diversity (Cipriani et al., 1999; Katzir et al., 1996). The abundance and distribution of SSR markers throughout a genome was shown in rice (Wu and Tanksley, 1993), in soybean (Akkaya et al., 1995), and in grape (Thomas and Scott, 1993). SSR markers can serve as anchor markers between different genetic linkage maps within specific crops due to their high level of reproducibility and polymorphism (Beckmann and Soller, 1990). In many cases SSR markers can be transferred between genetic maps derived from different populations (Westman and Kresovich, 1998) and in comparative mapping (Byrne et al., 1996). Eighty percent transferability of microsatellites has been reported among species of Myrtaceae, Poaceae and Pinus, with $20 \%$ transferability across unrelated genera (Gupta and Varshney, 2000).

SSR markers have been developed in different laboratories for research on Malus species (Gianfranceschi et al., 1998; Guilford et al., 1997; Hokanson et al., 1998). Malus spp. genomes are abundant in microsatellites, and the frequency of occurrence of di- and tetranucleotides is comparable with other plant species (Guilford et al., 1997). In a 'Golden Delicious' genomic library, the most abundant di-nucleotide was (GA) 10 and in general tri- and tetra-nucleotides were less frequent (Hokanson et al., 1998). Gianfranceschi et al.

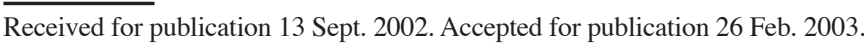

(1998) reported 8.2 alleles per locus and on average 78\% heterozygosity for 17 sets of SSR primers they developed in apple. Due to a high level of heterozygosity, as few as three selected SSR primers were sufficient to distinguish 21 apple cultivars (Guilford et al., 1997). Ciprani et al. (1999) reported that $88 \%$ of the microsatellites primers they developed for peach [Prunus persica (L.) Batsch] showed polymorphism and each had as many as two to four alleles. The same report indicated that $59 \%$ of the Prunus microsatellites gave correct amplification in a cross-species (almond, apricot, plum, sweet and sour cherry ) survey. Yamamoto et al. (2001) reported 79 alleles from seven Malus SSR primers detected in Pyrus. The flanking regions for selected SSRs were highly conserved in different pear accessions. However, fragment sizes were different between apple and pear and among pear cultivars.

The purpose of this study was to map 41 SSRs developed from apple by different laboratories on the 'Rome Beauty ' $\mathrm{x}$ 'White Angel' linkage map (Hemmat et. al., 1994), and to identify corresponding linkage groups on four other apple maps. Flanking primers developed for apple were also evaluated on two pear cultivars.

\section{Materials and Methods}

Plant matrerial. Three apple populations: 'Rome Beauty' x 'White Angel' (RB x WA) (56 trees), 'Wijcik McIntosh' x NY 75441-58 (WM x NY-58) (60 trees), and 'Liberty' X 'Golden Delicious' (Li x GD) (55 trees) and one pear population, 'Bartlett' $x$ NY 10353 (Ba X NY) were used in our study of genetic mapping of microsatellites. Plant material for the pear population was provided by Richard Bell, USDA, Kearneysville, W. Va.

Genetic linkage maps of 'Rome Beauty'x 'White Angel', (Hemmat, etal., 1994) and 'Wijcik McIntosh'x NY75441-58, (Conneret al., 1997) were used for linkage analysis and mapping of the SSRs. A Microsoft Excel macro, QUIKMAP, designed by Weeden, and Barnard (1994) to calculate the frequency of the matches between 
Table 1. SSR primers ${ }^{\mathrm{z}}$ used for mapping on genetic linkage map of three apple populations and one pear population.

\begin{tabular}{|c|c|c|}
\hline Primer name & Primer forward sequence & Primer reverse sequence \\
\hline${ }^{* \mathrm{SSR}^{\mathrm{CO}}}$ & CGC TTC GAA AAC CTC AAG AGA TCA TC & GAC GCC ATA CCC ATT GAC GA \\
\hline CH01B12 & CGC ATG CTG ACA TGT TGA AT & CGG TGA GCC CTC TTA TGT GA \\
\hline CH01E12 & AAA CTG AAG CCA TGA GGG C & TC AAT TCA CAT GAG GCT G \\
\hline CH01 G12 & CCC ACC AAT CAA AAA TCA CC & TGA AGT ATG GTG GTG CGT TC \\
\hline $\mathrm{CH} 01 \mathrm{H} 01$ & GAA AGA CTT GCA GTG GGA GC & GGA GTG GGT TTG AGA AGG TT \\
\hline $\mathrm{CH} 01 \mathrm{H} 10$ & TGC AAA GAT AGG TAG ATA TAT GCC A & AGG AGG GAT TGT TTG TGC AC \\
\hline CH02B10 & CAA GCA AAT CAT CAA AGA TTC AAG & CAA GTG GCT TCG GAT AGT TG \\
\hline CH02B12 & GGC AGG CTT TAC GAT TAT GC & CCC ACT AAA AGT TCA CAG GC \\
\hline $\mathrm{CH} 02 \mathrm{CO} 6$ & TGA CGA AAT CCA CTA CTA ATG CA & GAT TGC GCG CTT TTT AAC AT \\
\hline CH02D12 & AAC CAG ATT TGC TTG CCA TC & GCT GGT GGT AAA CGT GGT G \\
\hline $\mathrm{CH} 01 \mathrm{~F} 02$ & ACC CAC ATT AGA GCA GTT GAG G & CTG GTT TGT TTT CCT CCA GC \\
\hline *GD-6 & ATT TTG GAA CAC ACA CTC CCT CAG A & GAC GCC ATA CCC ATT GAC GAT T \\
\hline *GD-12 & TTG AGG TGT TTC TCC CAT TGG A & CTA ACG AAG CCG CCA TTT CTT T \\
\hline *GD-96 & CGG CGG AAA GCA ATC ACC T & GCC AGC CCT CTA TGG TTC TCA GA \\
\hline GD-100 & ACA GCA AGG TGT TGG GTA AGA AGG T & TGC GGA CAA AGG AAA AAA AAA AGT G \\
\hline$*$ GD-103 & CGG CGA GAA AAA AAA ACA ATG & GGA TAA CCG TCC CCC TCT TC \\
\hline *GD-112 & GCT GCG AGC GAA CCT GAA & ACC TGA ATC CGA CCC CTT TTC \\
\hline *GD-126 & ATT TCG TTG CTG TGC TCG TC & AAC CTC CTT CAT TAC ATT CCA CTC T \\
\hline *GD-127 & CTT TCG GAT GCC TTT TGC T & GCT TTC GGA TTC TTC GTC TTC \\
\hline *GD-136 & CGG CGA GAA AAA AAA ACA ATG & GTG GAT AAC CGT CCC CTC TTC \\
\hline GD-142 & GGC ACC CAA GCC CCT AA & GGA ACC TAC GAC AGC AAA GTT ACA \\
\hline *GD-144 & GCA AAC ATA AAA CCC ATC ATT ACC & GTA GCC CTC AAA GAA CTC CTT GT \\
\hline GD-147 & TCC CGC CAT TTC TCT GC & AAA CCG CTG CTG CTG AAC \\
\hline *GD-153 & AAG CCC TAT CTA TTC TTC ACA CAC A & ССТ ТTC ССС СТС АСА CAA C \\
\hline *GD-154 & CGT GGA GGA AGA GAA AGA GGC & GCAGAG TGA CCC TGA AGA CAA \\
\hline *GD-158 & CAA CCA CTC AGA ATG ACA CCA AC & GAG AAA GAC GAT GGC TAC GAG ATT \\
\hline$*$ GD-161 & TTT GGG TCC CTG AGA GTC CAG TG & GTC GTT TGA AAT CCA TCC CTC CAT \\
\hline GD-162 & GAG GCA AGT GAC AAA GAA AGA YG & AAA ATG TAA CAA CCC GTC CAA GTG \\
\hline *NZ-02b1 & CCG TGA TGA CAA AGT GCA TGA & ATG AGT TTG ATG CCC TTG GA \\
\hline *NZ-03c1 & GCT CTC ATC TTC ACA GAT AA & AGA CCC GGA AAA TTC TAT \\
\hline *NZ-04h11 & CTT CCA TCG AGATTG CATC ATA & CGA ATT GAG AGG TCG TCG TT \\
\hline *NZ-05g8 & CGG CCA TCG ATT ATC TTA CTC TT & GGA TCA ATG CAC TGA AAT AAA CG \\
\hline *NZ-17e6 & AAC ACG CCA TCA CAC ATC & CTG TTT GCT AGA AGA GAA GTC \\
\hline NZ26c6 & GAC GAA GAA CTC GCC GGC GC & CGA GGA CCA ACC CAC ACA CAA \\
\hline *NZ-28f4 & TGC CTC CCT TAT ATA GCT AC & TGA GGA CGG TGA GAT TTG \\
\hline *UK-MS11H08 & TAT TAG TGC TTG ACA GAC & CAG CAT GAT TAG TAG TTT AG \\
\hline *UK-MS14B04 & CCT TAA GAA TCA TGT GAT & ACT AAT GGC ACA AAG ATT GT \\
\hline *UK-MS14H03 & CGC TCA CCT CGT AGA CGT & ATG CAA TGG CTA AGC ATA \\
\hline *UK-MS02A01 & CTC CTA CAT TGA CAT TGC AT & TAG ACA TTT GAT GAG ACT G \\
\hline *UK-MS01A03 & AGC AGT ATA GGT CTT CAG & TGC GTA GAT AAC ACT CGA T \\
\hline *UK-MS01A05 & GGA AGG AAC ATG CAG ACT & TGA TGT TTC ATC TTC ACA \\
\hline *UK-MS02H06 & AGA CGA ACA CAA GTA GTA & CTA ATA CAG TCA TAC AG \\
\hline
\end{tabular}

zPrimers identified by CH were developed at the Institute of Plant Sciences, Swiss Federal Institute of Technology, Universitätstrasse 2, CH-8092 Zürich, Switzerland, GD primers were developed at the USDA/ARS-Plant Genetic Resources Unit, Cornell University, Geneva, NY. Primers identified as NZ, were developed at the Horticulture and Food Research Institute of New Zealand. Primers designated as UK, were developed at the Plant Genetics and Development, Horticulture Research International, Wellesbourne in the United Kingdom and sequences generously supplied by Dr. G. King.

*Primers used on pear population.

the marker loci and the SSR loci, was used to identify the position of the SSR loci on the linkage maps. The identified SSRs for each linkage group served as anchor points to find corresponding linkage groups in other populations.

DNA ISOLATION AND PCR AMPLIFICATION OF SSR LOCI. Genomic DNA was extracted from very young leaf tissue using CTAB buffer according to Saghai-Maroof et al. (1984) and a modification of the method by Doyle and Doyle (1990). A total volume of $25 \mu \mathrm{L}$ reaction mixture containing $2.5 \mu \mathrm{L} 10 \times$ buffer, $1.5 \mu \mathrm{L}$ of $2.5 \mathrm{~mm}$ of each $\mathrm{dNTP}, 10 \mu \mathrm{M}$ of each primer and $0.5 \mathrm{U}$ Taq polymerase (Promega Corp., Madison, Wis.) and $200 \mathrm{ng}$ DNA was subjected to PCR amplification in a thermocycler (PTC-100; MJ Corp., Watertown, Mass.). Cycling parameters for amplification of SSR included an initial cycle of $60 \mathrm{~s}$ at $94^{\circ} \mathrm{C}$ followed by 40 cycles of $60 \mathrm{~s}$ at 94 ${ }^{\circ} \mathrm{C}, 120 \mathrm{~s}$ at the annealing temperature (between 49 to $59^{\circ} \mathrm{C}$ ), 120 s at $72{ }^{\circ} \mathrm{C}$ and a final cycle of $8 \mathrm{~min}$ at $72^{\circ} \mathrm{C}$.

SOURCES OF PRIMER SEQUENCES. In total, 41 SSR primer sets were used on apple and 27 sets on pear (Table 1). Primers identified as GD were developed at the USDA-ARS, Plant Genetic Resources Unit, Cornell University, Geneva, N.Y. (Hokanson et al., 1998). Primers designated $\mathrm{CH}$ were developed at the Institute of Plant Sciences, Swiss Federal Institute of Technology, Zurich, Switzerland 


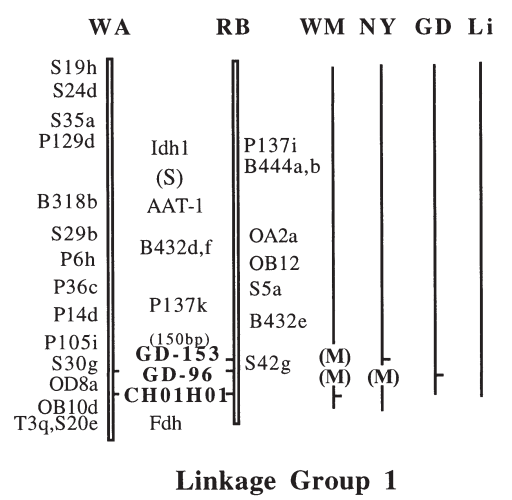

Linkage Group 1

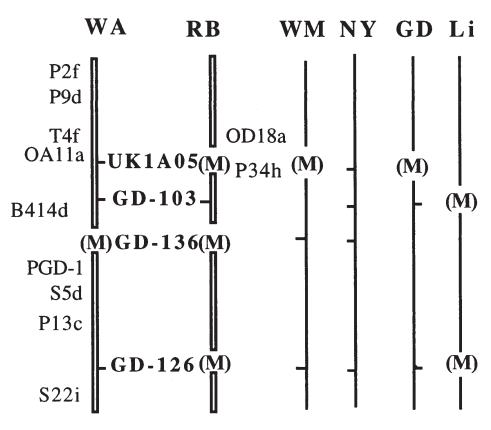

Linkage Group 2

(Gianfranceschi et al., 1998). Primers identified as NZ were developed at the Horticulture and Food Research Institute of New Zealand, Auckland (Guilford et al., 1997). Seven primer sets, designated as UK, were developed in the United Kingdom and generously supplied by G. King (Plant Genetics and Development, Horticulture Research International, Wellesbourne).

ELECTROPHORESIS OF THE AMPLIFICATION PRODUCTS. PCR products were separated on NuSieve GTG agarose gel (FMC Bioproducts, Rockland, Maine) using $1 \times$ TBE buffer, at $\approx 2.6$ $\mathrm{V} \cdot \mathrm{cm}^{-1}$. The gels were run $\approx 1.5$ to $2 \mathrm{~h}$. The concentration of agarose used in the gels varied between $3.5 \%$ to $4.25 \%$ depending on the size of the amplified fragment to be resolved. Gels were stained with ethidium bromide and photographed under UV $(\lambda-302 \mathrm{~nm})$ light using

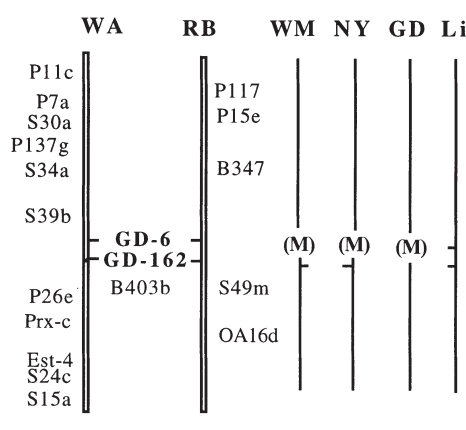

Linkage Group 5

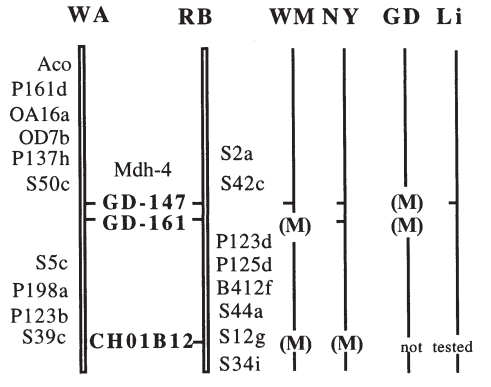

Linkage Group 4

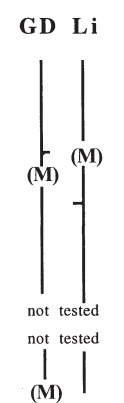

(1)

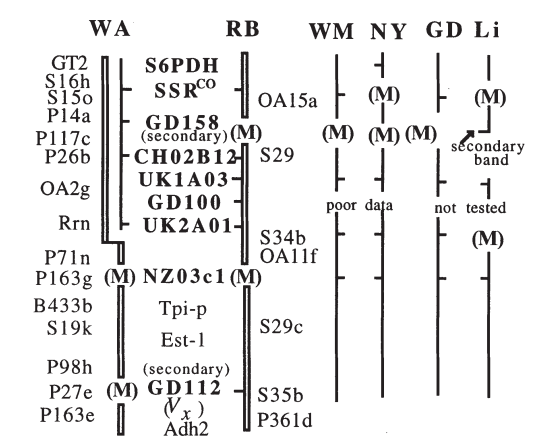

Linkage Group 6

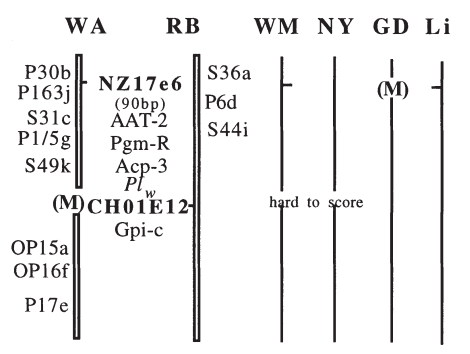

Linkage Group 7

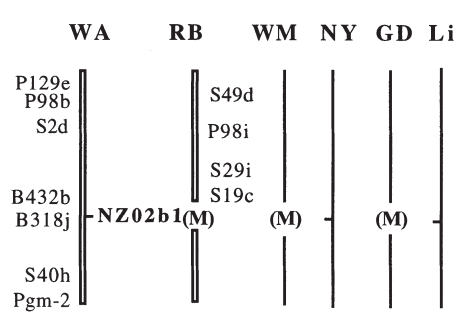

Linkage Group 9

\section{Results}

Polymorphism. An initial comparison of agarose (2\% to $4 \%$ NuSieve or equivalent) and denaturing sequencing gels indicated that most polymorphisms resolved on the sequencing gels also could be resolved on agarose gels. Thus the present analysis was performed solely on agarose gels. All primer sets except UK11H08 gave clear polymorphisms in at least one of the apple populations. As expected, 'White Angel' produced the most segregating bands ( $84 \%)$. NY 75441 58 produced the second most (72\%), and the remaining four cultivars all displayed about $60 \%$ segregating bands for the SSR loci (data not presented).

Of the 41 primer sets producing amplified product, all generated fragments near the predicted size except for GD-127 and GD-103. The GD-127 primer set did not amplify a $120 \mathrm{bp}$ fragment in the RB $\times$ WA cross, but produced the expected fragment in the other two apple populations as well as in the pear population. For GD-103 an $80 \mathrm{bp}$ fragment was polymorphic in the RB $x$ WA progeny rather than the expected 108 bp fragment. Several SSR primers, including GD-6, GD-103, GD-153, and GD-158 generated additional fragments, some of which were polymorphic and assorted independently of the primary polymorphism. Increasing the annealing temperature in these cases often led to the loss of all amplification products rather than loss of just the unexpected fragments. Finally, even after multiple attempts, certain SSR primers sets failed to generate fragments in specific crosses: UK2H06 and NZ5g8 in WM x NY-58, and GD-161 in Li x GD.

Distribution OF SSR MARKERS ON THE STANDAD

Fig. 1. Distribution and approximate position of SSR markers on the genetic linkage map of 'White Angel' (WA), 'Rome Beauty' (RB), and corresponding linkage groups in 'Wijcik MacIntosh' (WM), NY 75441-58 (NY-58), 'Golden Delicious' (GD), and 'Liberty' (Li) apple cultivars. Mapped SSR markers are noted by a horizontal bar on the linkage group. The approximate location of the markers or gene not segregating in the test population are noted by $(\mathrm{M})$ or gene symbols in parentheses. SSR markers mapping on more than one linkage group are noted by the size of the bands. 


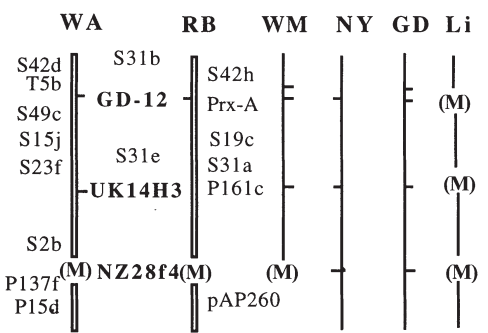

Linkage Group 10
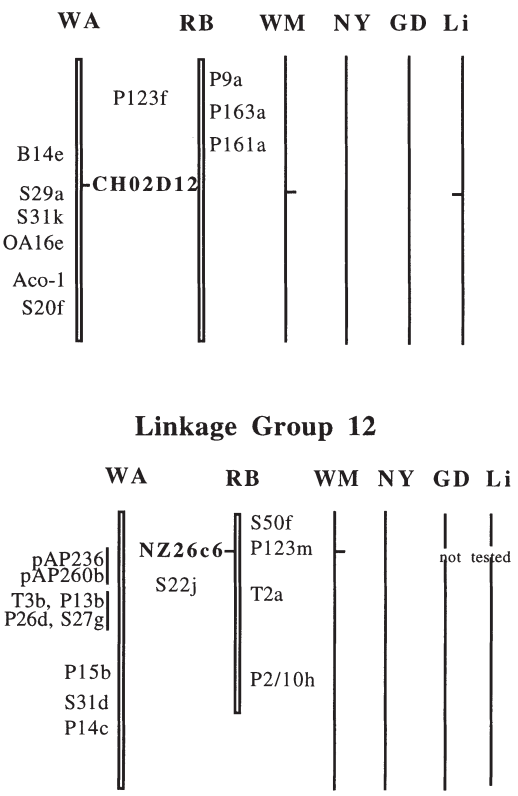

Linkage Group 15

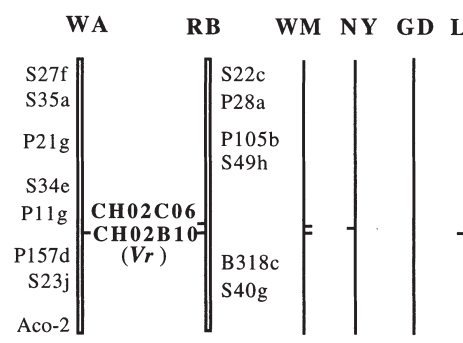

Linkage Group 11

these markers, as a group, segregated independently of the remaining markers defining LG 6. This suggests that at least one chromosomal rearrangement has occurred between Asian Malus species such as M. sargentii and $M$. sieboldii (from which 'White Angel' has been derived) and $M . \times$ domestica.

2) In both RB $x$ WA and Li $x$ GD, GD-6 mapped to LG 5. GD-6 was closely linked to GD-162 in both the former progeny, and GD-162 mapped to LG 5 in WM $x$ NY-58. However, in WM x NY-58, GD-6 mapped to LG2. Thus, we suggest that we scored a nonhomologous GD-6 polymorphism in the WM x NY-58 progeny.

In some cases SSR markers identified a homologous linkage group or reassigned homologous linkage groups from those previously reported (Hemmat et al., 1994).

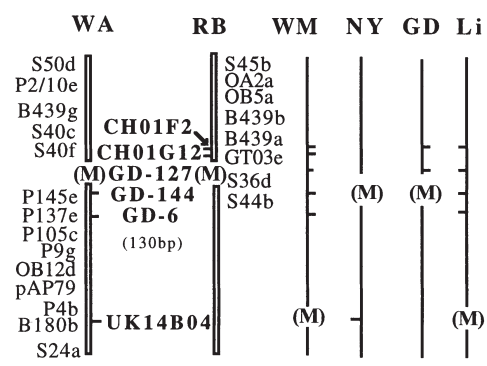

GD-96 and GD-153 identified a homologous linkage group for WALG 1. CH01H01 also mapped on the bottom of LG 1 and identified LG 17 as continuation of LG 1. Of four SSR markers mapped on LG 2, GD-103 and UK1A05 were particularly useful in identification of a homologous LG for WA LG 2. The $V_{x}$ scab resistance gene from R12740-7A was mapped through distal markers on LG 10 of 'Wijcik McIntosh', and this same region can be assigned to the bottom of LG 6 WA (Hemmat et al., 2002).

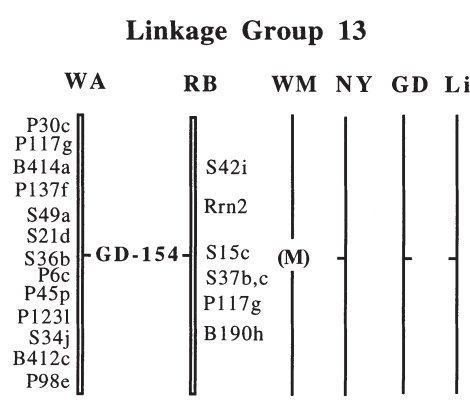

Linkage Group 16

In four cases, SSRs identified new or re-assigned linkage groups from those previously reported. For instance, GD-12 and UK14H03 were the most useful SSR markers for assigning RB LG 5 and WA LG 8 to the new LG 10. Among three SSRs mapped on LG 4, GD-147 and GD-161 were the most useful in identifying RB 16 as the homologous group for LG 4. GD-6 and GD-162 mapped to WA 5 and identified newly assigned RAPD markers for the new RB LG 5. The only SSR on LG 16, GD-154, identified RB $\mathrm{X}-3$ and additional RAPD markers as the new RB LG 16. NZ26c6 mapped on LG 15 of WM and displayed weak linkage to markers on RB LG 15.

In pear, we observed the same general pattern of

Fig. 1 (continued). Distribution and approximate position of SSR markers on the genetic linkage map of 'White Angel' (WA), 'Rome Beauty' (RB), and corresponding linkage groups in 'Wijcik MacIntosh' (WM), NY 75441-58 (NY58), 'Golden Delicious' (GD), and 'Liberty' (Li) apple cultivars. Mapped SSR markers are noted by a horizontal bar on the linkage group. The approximate location of the markers or gene not segregating in the test population are noted by $(\mathrm{M})$ or gene symbols in parentheses. SSR markers mapping on more than one linkage group are noted by the size of the bands.

LINKAGE MAP. All 41 SSR primer pairs tested produced markers that could be mapped in our populations (Fig. 1). At least one SSR was placed on 15 of the 17 linkage groups (LG) (Table 2). Clusters of SSRs were observed on LG 1, 2, 3, 4, 5, 6, and 13 (Table 2, Fig. 1). LG 3 and 6 were especially rich in SSR loci, possessing six and nine SSRs, respectively. UK2A01 and GD-100 on LG 6 displayed identical segregation patterns and appeared to be very tightly linked.

Comparative MaPPING. The SSR primer sets identified or confirmed homologous linkage groups in WM, NY-58, GD, and $\mathrm{Li}$ (Fig. 1). SSRs syntenic in WA or RB were also syntenic in the other cultivars except in two cases:

1) UKA103, UK2A01, GD-100, and SSR ${ }^{C o}$ [marker for columnar habit (Co) Hemmat et al., 1997] all mapped on LG 6 of RB, and most mapped on LG 6 of WM, NY-58, and GD. However, in WA segregation. In most cases, a 2 to $3{ }^{\circ} \mathrm{C}$ lower annealing temperature was required for amplification of products. From 27 SSRs tested on 'Bartlett' and NY 10353-85001, 22 were polymorphic. UK2H06, UK11H08, NZ4H11 and SSR ${ }^{\text {co }}$ did not amplify a fragment of the expected size and UK1A03 was monomorphic.

Comparison between Hemmat ET AL. (1994) ANd MaliepaARd ET AL. (1998) MAPS. Although researchers have numbered their linkage groups differently, the anchor loci should permit convenient inter-conversion and comparison of these two maps. We also included the map of Gianfranceschi et al. (1998) which is based on a map of 'Prima' X 'Fiesta' (Pr X Fi) (Maliepaard et al., 1998). We identified markers (isozyme, RFLP, and SSR) in 15 linkage groups that could serve as anchor markers for comparison between the maps (Table 3). For LG 1, Aat-1 and the self-incompatibility locus ( $S$ ) from 'White Angel' and CH01H01 identified LG 17 as corresponding linkage group. We mapped pAP79 on LG 13 and $M e$ on LG 14 of RB $\times$ WA. These two markers were mapped on LG 2 of Fi. We mapped CH01 B12 on LG 4 and CH01F2 on LG 13 of $\mathrm{RB}$, while these two markers were mapped on one linkage group in 'Iduna' and 'A679/2' ('I'-5 and 'A'-11) (Gianfranceschi et al., 1998). CH01B12 was tightly linked to the markers $S 12 \mathrm{~g}$ and S34i on RB 4. Pgm-2 and NZ2b1 both mapped on LG 9 of WA, while Pgm-2 mapped to the bottom of LG 7 and NZ2b1 on LG 
Table 2. Distribution of SSR markers on different linkage groups of 'Rome Beauty' $x$ 'White Angel' population.

\begin{tabular}{|c|c|c|c|}
\hline \multirow{2}{*}{$\begin{array}{l}\text { Linkage } \\
\text { group }\end{array}$} & \multirow[b]{2}{*}{ SSR } & \multicolumn{2}{|c|}{ Segregating fragments } \\
\hline & & 'White Angel' & 'Rome Beauty' \\
\hline 1 & GD-96 & 162,166 & 167,176 \\
\hline 1 & GD-153 & None & 150 \\
\hline 2 & UK1A05 & 180 & 80,210 \\
\hline 2 & GD-103 & 85 & 82,108 \\
\hline 2 & GD-136 & None & None \\
\hline 2 & GD-126 & 260 & None \\
\hline 3 & NZ04H11 & 190 & 220 \\
\hline 3 & GD-153 & 200 & 230 \\
\hline 3 & GD-158 & 145 & 160 \\
\hline 3 & NZ-05g8 & None & 120 \\
\hline 3 & UK2H06 & 210 & None \\
\hline 3 & GD-142 & 142,144 & 135,142 \\
\hline 4 & GD-147 & 128 & 132,142 \\
\hline 4 & GD-161 & 190 & 210 \\
\hline 4 & $\mathrm{CH} 01 \mathrm{~B} 12$ & None & 170 \\
\hline 5 & GD-6 & 145 & 180,220 \\
\hline 5 & GD-162 & 219,236 & 227,240 \\
\hline 6 & S6PDH & None & None \\
\hline 6 & $\mathrm{SSR}^{\mathrm{Co}}$ & 150,170 & 185 \\
\hline 6 & GD158 & 135 & None \\
\hline 6 & CH02B12 & 145 & 165 \\
\hline 6 & UK1A03 & 200 & 180 \\
\hline 6 & GD-100 & 228,242 & 228,236 \\
\hline 6 & UK2A01 & 170,200 & 230 \\
\hline 6 & NZ03C1 & None & None \\
\hline 6 & GD-112 & None & 200 \\
\hline 7 & CH01E12 & None & 195 \\
\hline 7 & NZ17e6 & 90 & None \\
\hline 9 & NZ02b1 & None & None \\
\hline 10 & GD-12 & 170,200 & 152,154 \\
\hline 10 & UK14h03 & 160 & 110,150 \\
\hline 10 & NZ28F4 & None & None \\
\hline 11 & $\mathrm{CH} 02 \mathrm{~B} 10$ & 115,160 & 120,130 \\
\hline 12 & CH02D12 & 180 & None \\
\hline 13 & $\mathrm{CH} 01 \mathrm{~F} 2$ & None & 265 \\
\hline 13 & CH01G12 & None & 150,190 \\
\hline 13 & GD-127 & None & None \\
\hline 13 & GD-144 & 220 & 210 \\
\hline 13 & GD-6 & 80 & None \\
\hline 13 & UK14B04 & 280 & None \\
\hline 15 & NZ26c6 & None & 100 \\
\hline 16 & GD-154 & 175,200 & 180,95 \\
\hline
\end{tabular}

15 of Pr x Fi. NZ28f4, UKH03 and Prx-A all mapped on the new LG 10 of RB $x$ WA, while UK14H03 and Prx-A mapped on LG 3 and NZ28f4 on LG 12 of Pr $x$ Fi.

\section{Discussion}

We have shown that SSR markers are useful in anchoring as well as confirming previously identified homologous linkage groups in the map of 'Rome Beauty' $x$ 'White Angel'. Certain SSR primer sets were more useful than others. Those most useful showed a high level of heterozygosity in the parents and did not generate fragments from additional loci. The combination of different kinds of markers in apple now permits good anchor loci to be identified for most of the linkage groups (Table 3).

Knowledge of map location for monogenic morphological traits and for disease resistance genes will provide better understanding and tools for genetic improvement. Availability of SSR markers on each linkage group provides better reference to monogenic traits such as resistance to powdery mildew (Podosphaera leucotricha) from 'White Angel' $\left(P l_{w}\right)$ (Batlle and Alston, 1996), red fruit skin color $\left(R_{f}\right)$ (Cheng et al.,1996) and fruit acidity $(M a)$ (Maliepaard et al., 1998). Linkage of an SSR marker to columnar habit (Co) (Hemmat et al., 1997) and close markers of the $V_{r}$ and $V_{r}$ scab resistance genes from R12740-7A apple (Hemmat et al., 2002), and the $V_{g}$ (race 7) scab resistance gene (Durel et al., 2000) will aid pyramiding of multiple genes for resistance.

The identification of an apparent chromosomal rearrangement in 'White Angel' (LG 6) suggests that we may expect to see changes in linkage relationships in pear and certainly in other Rosaceae genera. Some of the SSRs we examined should be useful for analyzing linkage conservation within the Maloideae. However, certain of the SSR primer sets will be of value only for fingerprinting or genetic analysis within Malus.

The clustering of SSR loci is interesting because the clusters often contain SSRs from different laboratories. On LG 3 and 6, SSRs from three and four different programs, respectively, are present. Certain regions of the apple genome must be particularly rich in microsatellite sequences. It is also interesting that the microsatellites tend to be more prevalent on the linkage groups that are better defined in both the American and European maps. All of the SSR polymorphisms detected in the 'Rome Beauty' $x$ 'White Angel' progeny could be located on the standard map, indicating the map covers most of the nuclear genome. However, $>80 \%$ of the SSRs mapped to the first 10 linkage groups defined, which also contain $\approx 90 \%$ of the expressed genes mapped in apple but relatively fewer $(\approx 75 \%)$ of the RAPD markers mapped. It would appear that these first 10 linkage groups either contain the bulk of the nuclear genome or most of the polymorphic sequences.

The high level of reproducibility of these SSR markers on six different apple cultivar maps allows us to use them as anchor points between maps from different laboratories. Using isozyme, RFLP and SSR markers, we identified 15 corresponding linkage group between our maps and those of Maliepaard et al. (1998).

However, there are some differences between the maps. We mapped both NZ2b1 and Pgm-2 on LG 9, while these were mapped on LG 7 and 15 of Maliepaard et al. (1998). We mapped Prx-A, UK14H03, and NZ28f4 onLG 10 of several different apple cultivars, yet these were mapped on LG 3 and 12 of Maliepaard et al. (1998). In two of our maps the SSR markers UK 14H03 and NZ28f4 are linked and belong to the same linkage group. These also linked to the SSR GD-12, which was closely linked to Prx-A on LG 10.

Another point of disagreement among the maps is between LG 2 of Pr x Fi and our corresponding linkage group. Gianfranceschi et al. (1998) mapped CH01B12 and CH01f2 on one linkage group, 'I'-5 x 'A'-11. We mapped pAP79 and CH01F2 on LG 13 corresponding to LG 2 of Fi and 'I'-5 X 'A'-11, respectively. However, we examined the possibility of linkage between CH01B12 and the markers on LG 13. We found a weak linkage (over $25 \mathrm{cM}$ ) between S45a and CH01B12. Maliepaard et al. (1998) also mapped Me on Fi 2. However, we mapped Me on LG 14 of RB. Neither the two SSRs (CH01B12 and CH01f2) nor isozyme Me were linked on one linkage group in our maps. In addition, three linkage groups in our map have closely mapped markers with no major gap. However, there is a $30 \mathrm{cM}$ gap between $\mathrm{Me}$ and pAP79 on LG 2 of Fi. Therefore, we preferred to err on the side of caution and did not use linkage weaker than $25 \mathrm{cM}$.

Success in amplification and polymorphism of Malus primers 
Table 3. Suggested anchor markers and comparison of the apple maps.

\begin{tabular}{|c|c|}
\hline Hemmat et al.( 1994) linkage groups & Maliepaard et al. (1998) linkage groups \\
\hline $1(S$, Aat $-1, F d h$, GD-153) & 17 , and 'I'-1 \& 'A'-12 \\
\hline $2(P g d-1, \mathrm{UK} 1 \mathrm{~A} 05, \mathrm{GD}-103)$ & 14 \\
\hline $3\left(R_{f}, I d h-2, \mathrm{NZ04H11,} \mathrm{GD-142)}\right.$ & 9 \\
\hline $4(M d h-4$, GD147, GD-161, CH01B12) & top of 'I'-5 \& 'A'-11 \\
\hline 5 (Cathodal Prx, Est-4, GD-6, GD-162) & 4 \\
\hline $6 \operatorname{Rrn} 1$, Adh, SSR ${ }^{\mathrm{Co}}, \mathrm{GD}-100, \mathrm{UK} 2 \mathrm{~A} 01$ & 10 \\
\hline $6 \mathrm{CH} 02 \mathrm{~B} 12$ & 10 and 'I'-14, \& 'A'-6 \\
\hline 7 (Aat-2, Lap-2, powdery mildew $P l_{w}$ resistance) & 8 \\
\hline $8\left(V_{f}, P g m-1\right.$, Tpi-c2 $)$ & 1 \\
\hline 9 NZ02b1 & 15 \\
\hline $9(P g m-2)$ & 7 \\
\hline 10 Anodal Prx, (GD-12, UK14H03) & 3 \\
\hline 10 NZ28f4 (GD-12, UK14H03) & 12 , and ' $\mathrm{D}$ '-12, \\
\hline $11\left(V_{r}, \mathrm{CH} 02 \mathrm{~B} 10, \mathrm{CH} 02 \mathrm{C} 06\right)$ & 'I'-8 \& 'A'-4 \\
\hline 12 (CH02D12) & 'I'-7 \& 'A'-3 \\
\hline 13 (pAP79, UK14B4, GD-144, CH01F2) & 2 and 'I' -5 \& 'A'-11 \\
\hline 14 (Me, Lap-4) & 2 \\
\hline 15 (pAP236, pAP260b, NZ26c6) & 16 \\
\hline 16 (GD-154, Rrn2) & ? \\
\hline
\end{tabular}

"All linkage groups, unless otherwise specified, are from 'Prima' x 'Fiesta' (Maliepaard et al., 1998). 'I' and 'A' represent 'Iduna' and 'A679/2' (Gianfranceschi et al., 1998).

in pear population $(81 \%)$, is an indication of conserved microsatellite flanking regions that exist between Malus and Pyrus as opposed to Cipriani et al. (2000) who found only $18 \%$ of the peach SSRs amplified in apple. Our results suggesting a high degree of transferability of microsatellite primers across the two genera are in agreement with Yamamoto et al. (2001) and Monte-Corvo et al. (2001). However, we do not know if these are homologs until the linkage map for pear is prepared. Identified microsatellites on Malus linkage groups can be utilized as anchor loci in comparative mapping among Malus species and between Malus and Pyrus.

\section{Literature Cited}

Akkaya, M.S., R.C. Shoemaker, J.E. Specht, A.A. Bhagwat, and P.B. Cregan. 1995 Integration of simple sequence repeat (SSR) DNA markers into a soybean linkage map. Crop Sci. 35:1439-1445.

Batlle, I. and F.H. Alston. 1996. Genes determining leucine aminopeptidase and mildew resistance from the ornamental apple 'White Angel'. Theor. Appl. Genet. 93:179-192.

Beckmann, J.S. and M. Soller. 1990. Towards a unified approach to genetic mapping of eukaryotes based on sequenced tagged microsatellite sites. Bio/Technology 8:930-932.

Byrne, M., M.I. Marquez-Garcia, T. Uren, D.S. Smith, and G.F. Moran. 1996. Conservation and genetic diversity of microsatellite loci in the genus Eucalyptus. Austral. J. Bot. 44:557-563.

Cheng, F.S., N.F. Weeden, and S.K. Brown. 1996. Identification of co-dominan RAPD markers tightly linked to fruit skin color in apple. Theor. Appl. Genet.93: 222-227.

Cipriani, G., G. Lot, W.-G. Huang, M.T. Marrazzo, E. Peterlunger, and R. Testolin 1999. AC/GT and AG/CT microsatellite repeats in peach [Prunus persica (L.) Batsch]: isolation, characterization and cross-species amplification in Prunus. Theor. Appl. Genet. 99:65-72.

Conner, P.J., S.K. Brown, and N.F. Weeden. 1997. Randomly amplified polymorphic DNA-based genetic linkage map of three apple cultivars. J. Amer. Soc. Hort. Sci. $122: 350-359$.

Doyle J.J. and J.L. Doyle. 1990. Isolation of plant DNA from fresh tissue. Focus 12:13-15.

Durel, C.E. 2000. Five new molecular maps. DARE (Durable Apple Resistance in Europe (D.A.R.E.) Nwsl. 3, p. 6

Gianfranceschi, L., N. Seglas, R. Tarchini, M. Komjanc, and C. Gessler. 1998. Simple sequence repeats for the genetic analysis of apple. Theor. Appl. Genet 96:1069-1076.

Guilford, P., S. Prakash, M. Zhu, E. Pikkerink, S. Gardiner, H. Bassett, and R. Foster.
1997. Microsatellites in Malus $\times$ domestica (apple): abundance, polymorphism and cultivar identification. Theor. Appl. Genet. 94:249-254.

Gupta, P.K. and R.K. Varshney. 2000. The development and use of microsatellite markers for genetic analysis and plant breeding with emphasis on bread wheat Euphytica 113:163-185.

Hemmat, M., N.F. Weeden, and S.K. Brown. 2002. Tagging and mapping scab resistance genes from R12740-7A apple. J. Amer. Soc. Hort. Sci. 127:365-370.

Hemmat, M., N.F. Weeden, P.J. Conner, and S.K. Brown. 1997. A DNA marker for columnar growth habit in apple contains a simple sequence repeat. J. Amer. Soc. Hort. Sci. 122:347-349.

Hemmat, M., N.F. Weeden, A.G. Manganaris, and D.M. Lawson. 1994. Molecular marker linkage map for apple. J. Hered. 85:4-11.

Hokanson, S.C., A.K. Szewc-McFadden, W.F. Lamboy, and J.R. McFerson. 1998. Microsatellite (SRR) markers reveal genetic identities, genetic diversity and relationship in a Malus $\times$ domestica Borkh. core subset collection. Theor. Appl. Genet. 97:671-683.

Katzir, N., Y. Danin-Poleg, G. Tzuri, Z. Karchi. U. Lavi, and P.B. Cregan. 1996 Length polymorphism and homologies of microsatellites in several Cucurbitaceae species. Theor. Appl. Genet. 93:1282-1290.

Maliepaard, C., F.H. Alston, G. Van Arkel, M. Brown, E. Chevreau, F. Dunemann, K.M. Evans, S. Gardiner, P. Guilford, A.W. van Heusden, J. Janse, F. Laurens, J.R. Lynn, A.G. Manganaris, A.P.M. den Nijs, N. Periam, E. Rikkerink, P. Roche, C. Ryder, S. Sansavini, H. Schmidt, S. Tartarini, J.J. Verhaegh, M. Vrielink-van Ginkel, and G.J. King. 1998. Aligning male and female linkage maps of apple (Malus pumila Mill.) using multi-allelic markers. Theor Appl. Genet. 97:60-73.

Monte-Corvo, L. Goulão, and C. Oliveira. 2001. ISSR analysis of pear and ability of molecular markers for clone discrimination. J. Amer. Soc. Hort. Sci. 126 $517-522$

Saghai-Maroof, M.A., K.M. Soliman, R.A. Jorgensen, and R.W. Allard, 1984. Ribosomal DNA spacer-length polymorphisms in barley. Mendelian inheritance chromosomal location and population dynamics. Proc. Nat. Acad Sci. USA 81 8014-8018.

Thomas, M.R. and N.S. Scott 1993. Microsatellite repeats in grapevine reveal DNA polymorphisms when analyzed as sequence tagged sites (STSs). Theor Appl. Genet. 86:985-990.

Weeden, N.F. and J. Barnard. 1994. QUIKMAP: A Microsoft Excel spreadsheet for making a quick comparison between markers in a linkage group and a new marker. N.Y. State Agr. Expt. Sta., Geneva, Computer Ctr. Tech. Rpt. 20-2.

Westman,A.L. and S. Kresovich. 1998. The potential for cross taxa simple-sequence repeat (SSR) amplification between Arabidopsis thaliana L. and crop Brassicas. Theor. Appl. Genet. 96:272-281.

Wu, K.S. and S.D. Tanksley. 1993. Abundance, polymorphism and genetic mapping of microsatellites in rice. Theor. Appl. Genet. 241:225-235.

Yamamoto, T., T. Kimura, Y. Sawamura, K. Kotobuki, Y. Ban, T. Hayashi, and N. Matsuta. 2001. SSRs isolated from apple can identify polymorphism and genetic diversity in pear. Theor. Appl. Genet. 102:865-870. 